\title{
PROGRESSIVE SPINAL MUSCULAR ATROPHY OF INFANTS (WERDNIG-HOFFMANN TYPE).
}

\author{
BY F. E. BATTEN, M.D. \\ AND \\ GORDON HOLMES, M.D.
}

THE following case was under our care in the Out-patient and the In-patient Departments of the National Hospital, and was exhibited at the Clinical Section of the Royal Society of Medicine on February 10, 1911, as a case of the Werdnig-Hoffmann type of the progressive spinal muscular atrophy of infants.

Some differences of opinion existed at first as to the diagnosis of the case, and it was originally suggested that the condition resembled that described by Oppenheim under the name "myatonia congenita." But though there was considerable loss of tone in the muscles, the marked paresis of the proximal muscles as compared with the distal, and the paralysis of the intercostal muscles pointed to a myelopathic rather than a myopathic disease.

\section{Clinical Bistory.}

The patient was the second of two children. The elder, a boy, died at the age of eight months of broncho-pneumonia; the parents described him as loose in his limbs and more or less similarly affected. No other instance of nervous disease was known in the family.

The patient was first seen in the Out-patient Department of the National Hospital in June, 1910, when she was 1 year 2 months old. She was born at the seventh month and had been delicate during the first four months of life, but she moved and kicked about as other children till she was about 6 months old. Then she seemed fat and healthy and was able to sit up, but was not encouraged to do so. At this age she was vaccinated and she cut her first tooth, but from this time onwards her parents noticed her becoming weaker and more helpless. At seven months she was able to say a few words. Between nine and twelve months she lost almost all power in her limbs and became unable to sit up, or to hold her head erect when it was unsupported.

When she was first seen she was a well nourished and fairly developed 
child; all her limbs were weak, especially at their proximal joints, and if she were placed in a sitting posture her back bent under her weight and her head hung backwards or to either side as drawn by gravity. Her limbs were flabby and toneless, but the atrophy of the muscles was obscured by a thick covering of subcutaneous fat. All the deep reflexes were absent. The movements of the eyes, face and tongue seemed unaffected, and the child was bright and intelligent. During the first few months that she was under observation she appeared to her mother to become a little stronger, but in October, 1910, at the age of eighteen months she was reported to be weaker, and then slight muscular contractures in the legs were noticed for the first time.

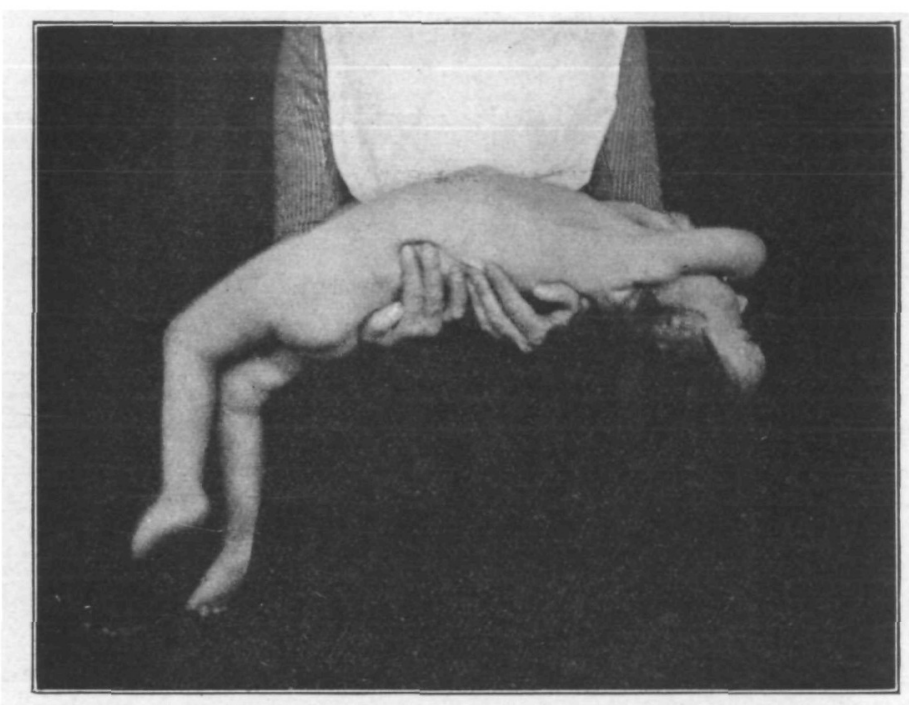

FIG. 1.-Photograph of cbild lying in nurse's arms showing her "limp" condition and her inability to raise her head or flex her legs.

She was admitted to hospital in January, 1911, at the age of one year and nine months. She was a bright and intelligent child and seemed to take an interest in all around her. She could talk, but it was difficult to understand what she said. She was easily upset and then cried in a normal manner, but evidently with very little breath, each few sobs being followed by an interval in which she made a few deep inspirations with considerable effort. The palpebral fissures were narrow and this gave the child the appearance which had been called "mongoloid," but apart from this there was nothing to suggest that she was a mongoloid idiot. The forehead had a central prominence over the frontal suture, but otherwise the head was normally shaped and measured $19 \frac{1}{2}$ inches in circumference. There was no sign of rickets : the incisors, canines and first molar teeth were present. 
The movements of the eyes, jaws, tongue and lips were quite normal, and the child swallowed without difficulty. She could also see and hear well, and obeyed commands as far as her muscular power permitted. Her optic dises were normal.

She could not raise her head from the pillow or even turn it from side to side. When placed in the sitting position the head fell backwards, or forwards, or to either side, the neck muscles being almost powerless. Her back also became bent, as there was no power in the trunk muscles either to fix or straighten the spine (figs. 1 and 2). When placed flat in

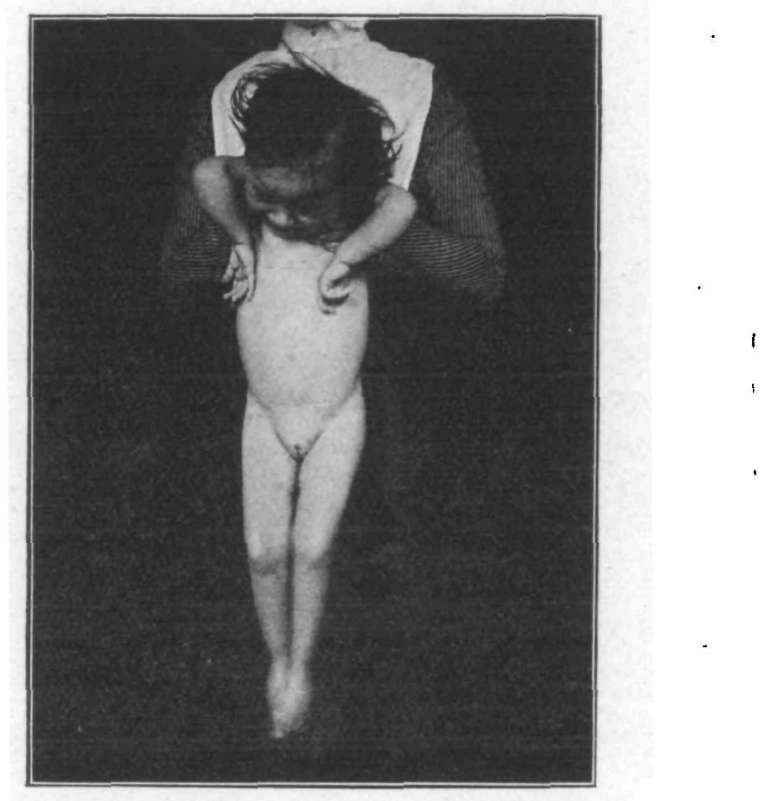

FIG. 2.-Photograph of child held up by a nurse; the head falls forward and the arms tend to "come away" from the trunk.

the bed, however, the spine became perfectly straight. The other trunk muscles were equally weak, with the exception of the diaphragm, which contracted vigorously. Her chest was long and narrow, and on each inspiration the intercostal spaces sank in, respiration being almost wholly diaphragmatic. The abdominal muscles though very weak were not completely paralysed.

The arms as a rule lay flexed at the sides of the chest, with the forearms pronated. She could make small movements with the fingers and grasp objects feebly, and could just flex and pronate the elbows, but not supinate the forearms. There was no power of movement however at the shoulderjoints. The lower limbs were almost powerless; practically no movement was possible at the hips or knees, but she had slight power of flexion and 
extension of the ankles and toes. The hamstrings were slightly contractured, so that the knees could not be fully extended.

The limbs were thin and their muscles much wasted, but this wasting was, to a considerable extent, obseured by subcutaneous fat. The muscles were also toneless, but the feet could not be brought over the shoulders nor the arms crossed behind her back, positions which are possible when there is marked hypotonia in a child.

Electrical examination showed complete loss of response to faradism in all the paralysed muscles, but the muscles of the hands responded fairly well. The child bore strong currents without evincing any signs of pain. In short, the child had an almost complete flaccid paralysis of all the muscles of the neck, trunk and limbs, the muscles of the face, eyes, and the diaphragm alone remaining normal.

Sensation to ordinary stimuli appeared to be unimpaired all over the body. The knee-jerks, ankle-jerks and the other deep reflexes were absent, but the sphincter functions were unaffected.

The child was removed from hospital in April, 1911, and died at home in the following January at the age of 2 years and 9 months.

The post-mortem examination was performed in the child's home fortyeight hours after death. There was then no rigor mortis present. The body appeared well nourished and so well covered with fat that the muscular wasting was scarcely apparent. When the skin was incised a thick layer of pale adipose tissue was found, and the muscles of the limbs and of the pelvic- and shoulder-girdles were so small and pale that it was, in certain instances, difficult to recognize them. Further, when they were cut across they were found to be soft and flabby, and the pallor was evidently due to the amount of fat they contained. This flabbiness and pallor of the muscles were more marked in the proximal segments of the limbs than in the distal. The rectus abdominis seemed to be less affected than most of the limb muscles, but the erector spinæ were extremely wasted and pale. The diaphragm was the only muscle which, in naked-eye appearance, approximated to the normal. Portions of a large number of muscles were taken for microscopical examination; they were fixed in Zenker's fluid, and embedded and cut in celloidin.

The larger nerve-trunks appeared unaffected to the naked eye; it could not be said they were smaller than normal. The brain and spinal cord also appeared normal, though the ventral roots of all segments of the cord were remarkably small and slender.

\section{Microscopical Examination.}

Nervous system.--The brain, spinal cord and several peripheral nerves were examined by Marchi's and by Weigert-Pal's methods, and in sections stained by hæmatoxylin and van Gieson's picro-fuchsin; several sections of the spinal cord were also stained by Nissl's method.

The Marchi method shows no evidence of either recent or old.degeneration 
in any part of the central or of the peripheral nervous system. The medullated fibres of the medulla oblongata and spinal cord stained well by the WeigertPal method, and there is no evidence of degeneration of any of the longer tracts. On the other hand, the staining of the medullated fibres in the grey matter is defective, and the medullated meshwork in the ventral horns is thinner than normal. The ventral roots in both their intra- and extramedullary courses are small and poorly stained, while the dorsal roots appear normal and stain well. The only definite change that is revealed by this method in the peripheral nerves is an apparent loss of fibres in some of the bundles, while a certain proportion of the fibres that remain are small and poorly stained.

Sections of the spinal cord stained by van Gieson's method show no inflammation or interstitial change, but the most striking feature apparent in sections of all regions is a paucity of cells in the ventral horns, and the small size of those cells which remain. In sections stained by Nissl's method the same remarkable deficiency of ventral horn-cells is even more striking. The small cells show no marked deviation from the normal; they appear like normal cells in miniature. The nuclei and nucleoli are situated centrally, and the chromophilic substance stains well.

All groups of ventral horn-cells are severely affected, but the posterolateral group in the cervical region is relatively best preserved. This deficiency of cells was confirmed by an enumeration of all those visible in a series of sections $10 \mu$ thick. In fifteen sections from the third lumbar segment, for instance, there was an average of only eight cells in the right ventral horn and 77 in the left, while in these fifteen sections only seven large cells; were counted, the others being all small. For the same segment of the normal cord, Bruce gives the average number of large cells as fortyseven, and nine small cells per section. Figs. 8, 9, 10 and 11, which are photographs and drawings of sections from the same segments of the cervical regions of the affected and of a normal cord, illustrate very well the deficiency in number and the diminution in size of the ventral horn-cells. The control is taken from the cord of a child, aged 10 months, and therefore considerably younger than the patient. Another normal cord from a child, aged 5, was also compared with that of our patient, and, although there is but little difference in the size and number of cells at these two ages, the comparison with the cord of the younger seems fairer, and this has been consequently reproduced.

The cells of the rest of the grey matter of the spinal cord appear normal in size and number; Clarke's column and the intermedio-lateral cells of the lateral horn seem large and extremely well developed in contrast with the cells of the ventral horns.

Muscles. - The muscles which were removed from the body and have been examined were the trapezius, the pectoralis major, the rectus femoris, the biceps femoris, the rectus abdominis, the erector spinæ, the flexors of the fingers and the diaphragm. 


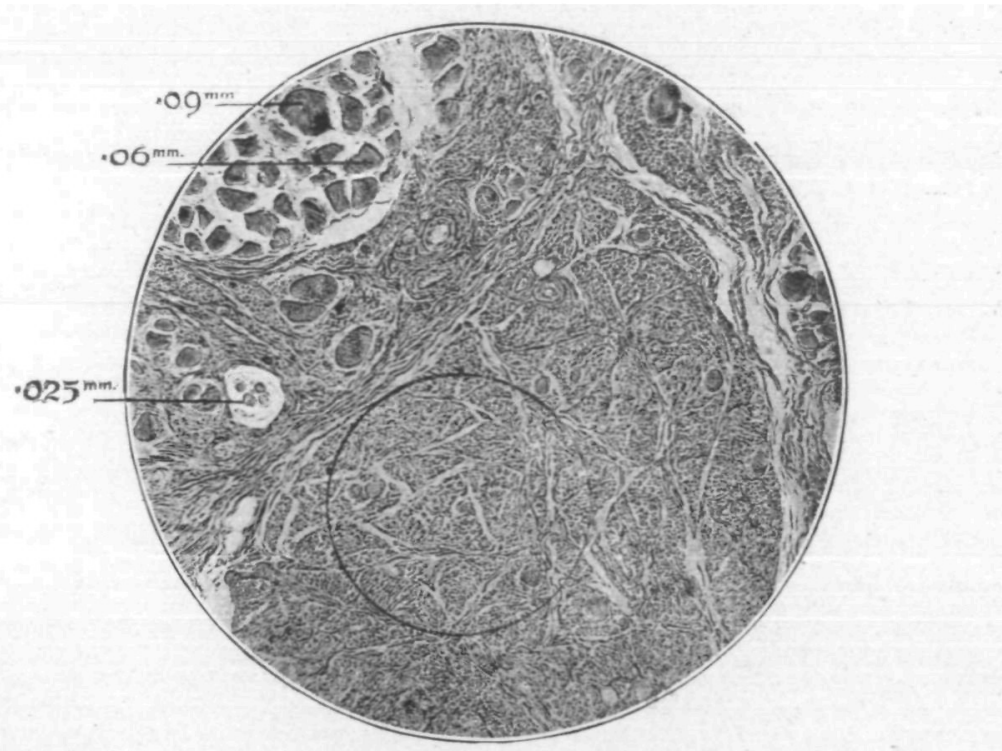

FIG. 3.-Transverse section of pectoralis major muscle, showing general structure of the muscle. A bundle of normal muscle-fibres is seen in the section; these measure from $0.04-0.9 \mathrm{~mm}$. in diameter. Scattered about the section are a few isolated fibres of normal size; but the majority of fibres have undergone extreme atrophy and measure $0.01 \mathrm{~mm}$. and less. A muscle-spindle containing four muscle-fibres is seen, the intra-fusal fibres measure $0.025 \mathrm{~mm}$., which is normal for these fibres. Magnification about 50 diameters.

The small circle indicates the area represented in the more highly magnified fig. 4.

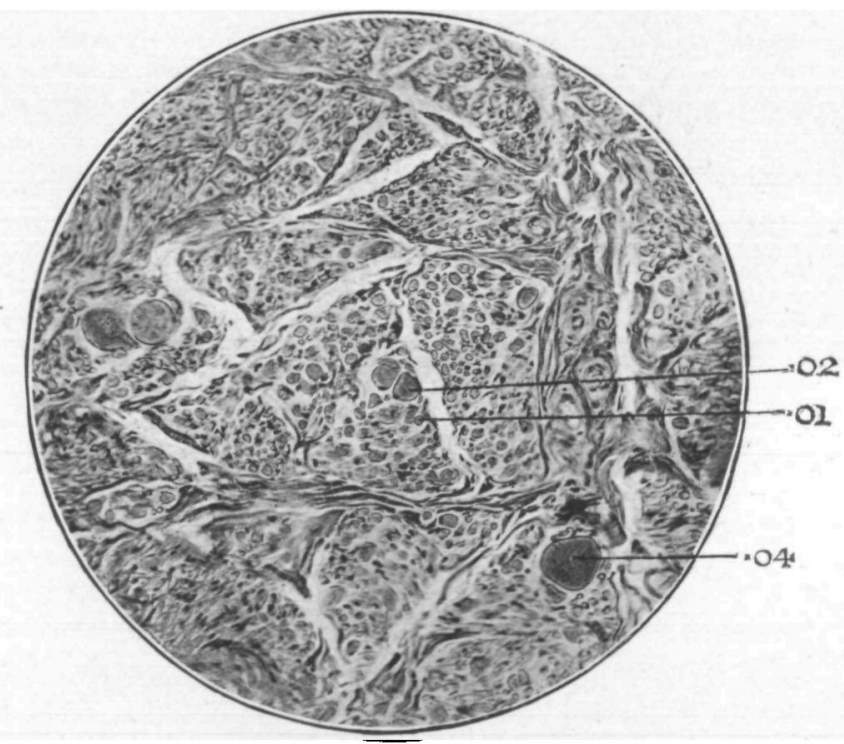

FIG. 4.-Transverse section of pectoralis major muscle under higher magnification. It shows a few comparatively large fibres about $0.04 \mathrm{~mm}$., but most of the fibres measure 0.01 mm. and some as little as 0.003 . Magnification about 150 diameters.

There is little or no increase of the connective tissue between the individual fibres. 
All these muscles have been cut in transverse and longitudinal sections, and stained by the van Gieson method.

The diaphragm alone of all the muscles examined shows no pathological change.

The changes in the affected muscles are strikingly similar, though they vary somewhat in the degree to which they affect the different muscles. All the muscles show a large number of atrophied or undeveloped muscle-fibres, with a few bundles of well-developed fibres. There is generally an increase in the amount of interstitial fat, and the connective tissue between the bundles is increased, although there is but little or no increase between the individual fibres. The latter feature stands out in rather striking contrast to the condition found in a myopathic muscle.

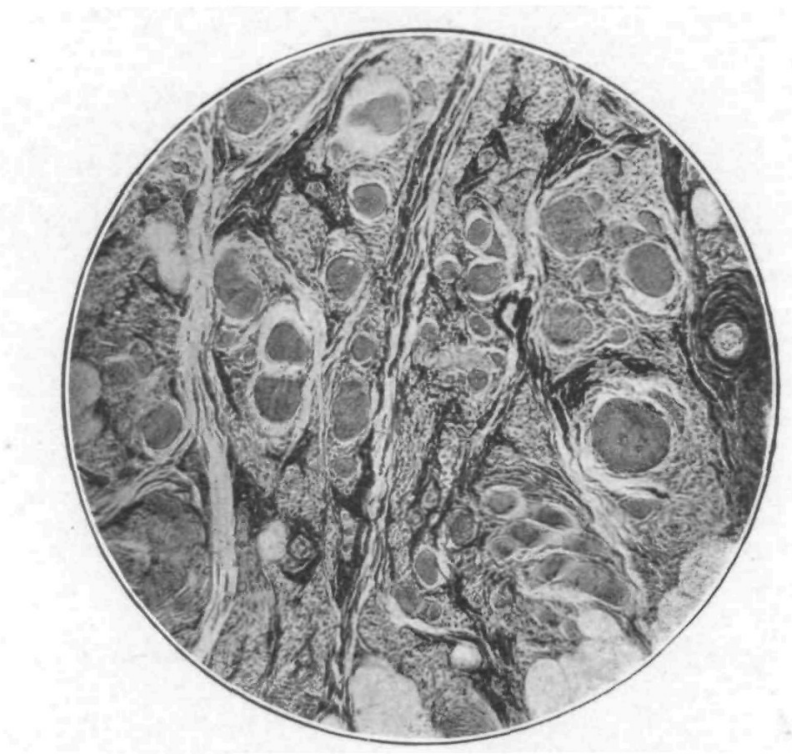

FIG. 5. - Transverse section of biceps femoris showing several large isolated fibres, some of which are surrounded by a number of atrophic fibres. There are numerous bands of connective tissue between the various muscle bundles. Magnification about 150 diameters.

A description of 'a section of the pectoralis major (fig. 3) will serve as an example of the changes present in all the muscles. In this muscle, bundles. of normal muscle-fibres having a polygonal shape can be seen measuring 06 to $09 \mathrm{~mm}$. in diameter (fig. 3). In close proximity to such normal bundles are bundles containing fibres of very small size, some measuring as little as $0025 \mathrm{~mm}$. Between these minute and atrophied fibres and the normal fibres all varieties of sizes are seen. Many of the small fibres are round or oval in outline, and not of the normal polygonal shape in cross-section. In some bundles in which almost all fibres are of small size a few large isolated fibres of a round or oval shape are present measuring $04 \mathrm{~mm}$. (fig. 4). The nuclei 


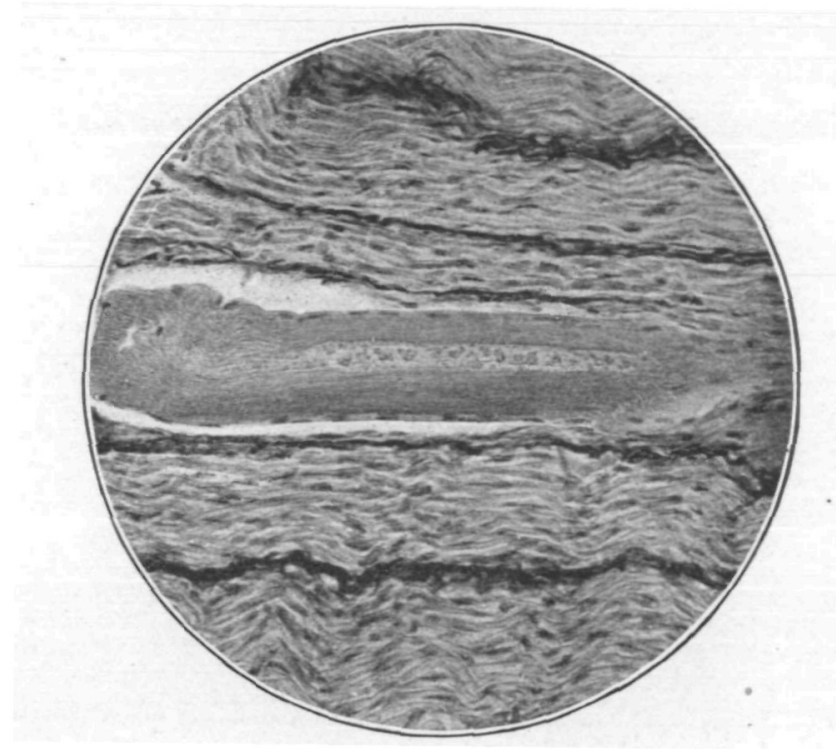

Fig. 6. -Longitudinal section of anterior tibial muscle showing degeneration of the centre of a large muscle-fibre. The neighbouring fibres have undergone extreme atrophy.

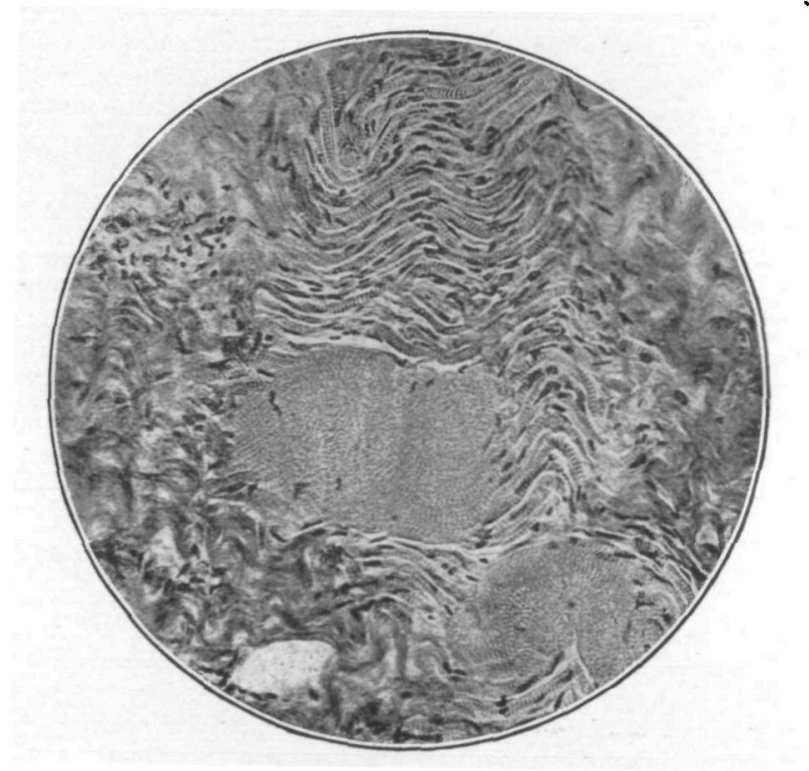

Fig. 7.-Section of rectus femoris muscle showing large fibres splitting into numerous fine fibres. The transverse striation of these small muscle-fibres is well preserved. 


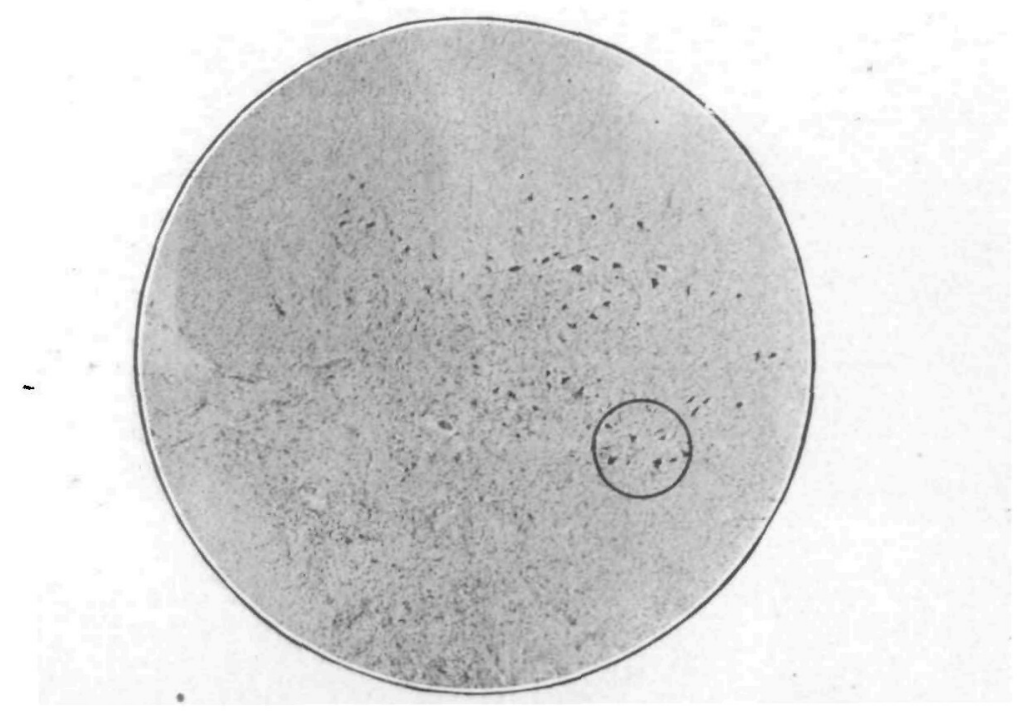

FIG. 8.-Photographs of the anterior horn of the cervical region of the spinal cord of a normal child, aged 10 montbs, to compare with the fig. 9 . The section bas been taken from the same segment in the cervical region, and has been similarly stained and photographed under the same magnification. The circle indicates the position of the cells seen under higher magnification in fig. 10.

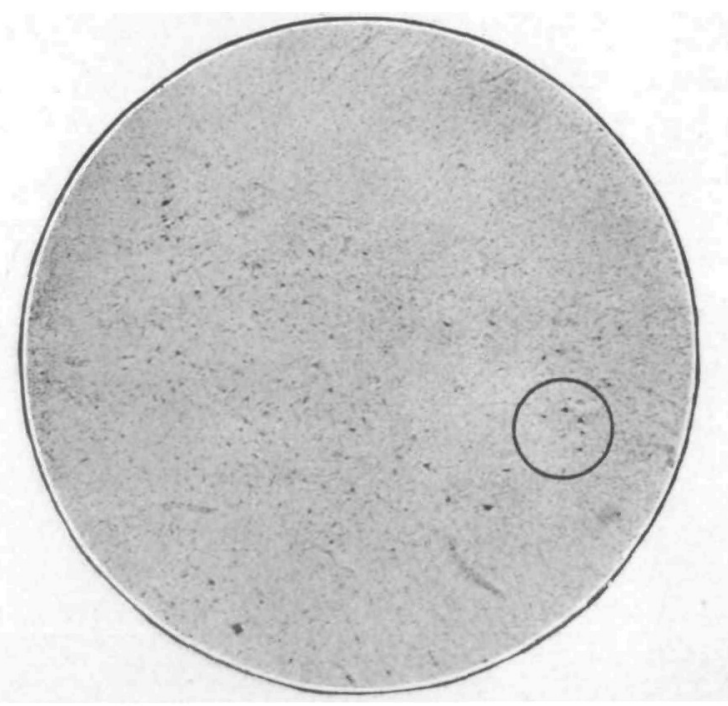

Fic. 9.-Anterior horn of the cervical region of the spinal cord showing the marked diminution in the number of the cells and the small size of those which still remain. The circle indicates the position of the cells seen under bigher magnification in fig. 11 . 


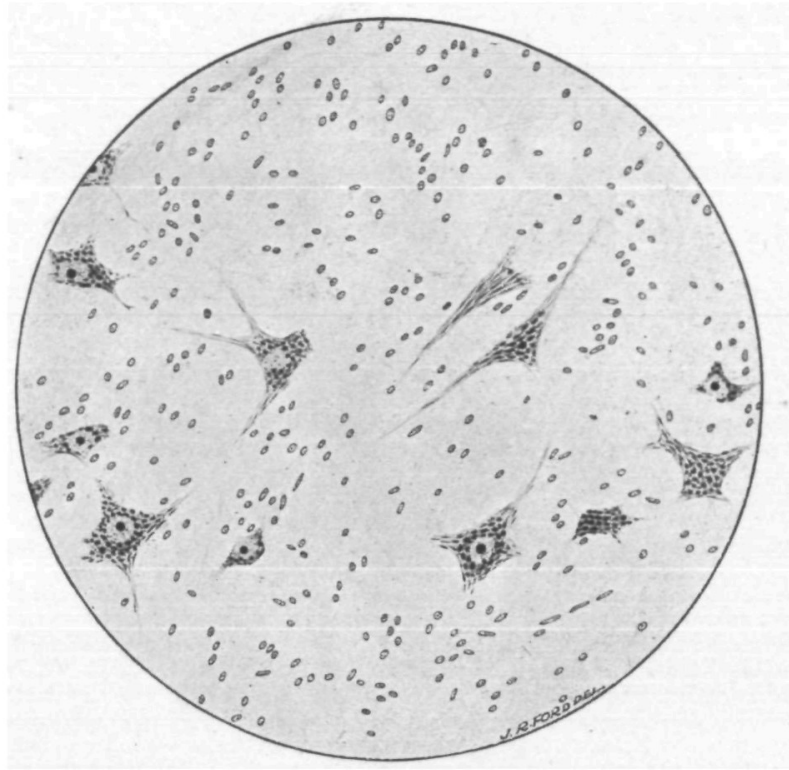

Fig. 10.-Normal cells of the anterior born under high power (child aged 10 months).

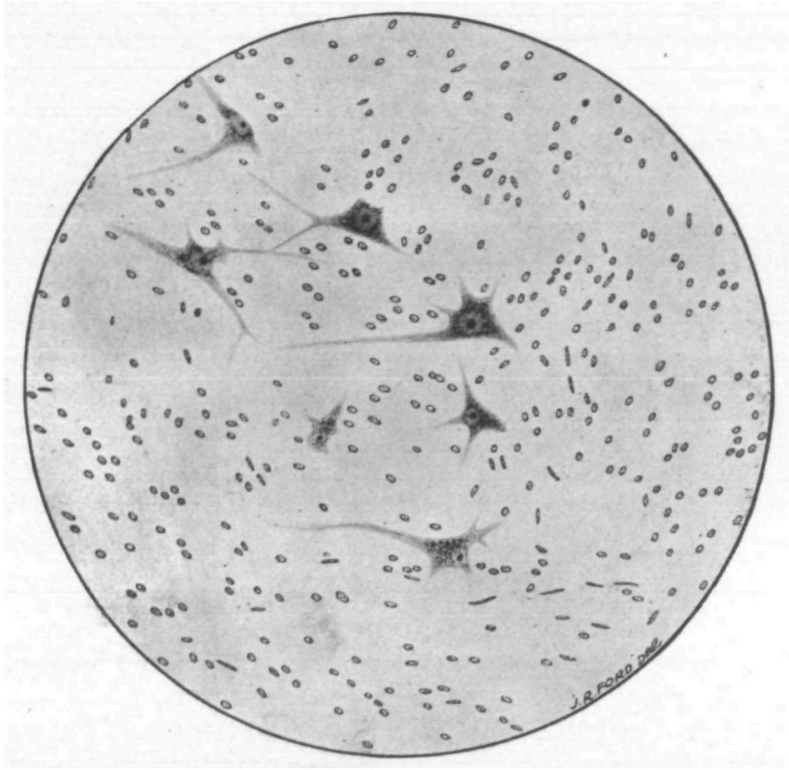

Fig. 11.-Cells of anterior horn under same magnification, showing their relatively small size and comparatively normal appearance.

II Figs. 10 and 11 bave been reproduced from drawings made from photographs taken under the same magnification.

(Drawings have been used in the place of photographs because of the impossibility of obtaining clear photographs of all the cells in the field.) 
of the muscle-sheaths are in most places normal; but in others are grouped together, and form a dark band surrounding certain muscle-fibres.

The connective tissues between the bundles is relatively increased owing to the smallness of the muscle-fibres (fig. 5).

Most of the fibres, even those which are extremely small, show normal transverse striation (fig. 7).

Some few fibres show central degeneration (fig. 6), and some sections show a splitting up of a large fibre into numerous fine fibres (fig. 7). There is an increase of the interstitial fat in some muscles; but it is not a marked feature. Numerous muscle-spindles can be seen, and their intra-fusal fibres appear of the normal size, viz., about ${ }^{\circ} 02 \mathrm{~mm}$. in diameter (fig. 3).

To summarize, it may be said that the majority of the muscle-fibres present the appearance of a simple atrophy, with but little, and often no, increase of connective tissue, or of nuclei between them. Targe and abnormally thick bands of fibrous tissue, and an excess of fat are, however, present in all the affected muscles. It seems possible that many of the small muscle-fibres may never have developed, as they show very little of such degenerative changes as are met with in conditions of progressive muscular atrophy in the adult. The presence of abnormally large fibres of cylindrical outline, and the occurrence of central degenerative changes, and of fission of some of the large fibres is, however, evidence that at least some of the fibres have undergone retrogressive changes similar to those which occur in both primary myopathic affections, and in the spinal muscular atrophies.

Commentary.-The clinical diagnosis of this case presented some difficulties. The marked loss of tone in the muscles, together with their weakness, suggested at first that the case was one of myatonia congenita. The onset of the weakness during the first nine months of life, and its progressive nature, however, indicated that the condition was similar to that described by Werdnig and by Hoffmann in young children under the title of "Progressive Spinal Muscular Atrophy of Infants." In support of the view that the condition was due to a spinal atrophy attention should be called to the facts: (1) That the intercostal and abdominal muscles were weak and paralysed; (2) that the paralysis of all the more proximately situated muscles was relatively greater than of the distal muscles, and (3) that the loss of tone-hypotonia-was not so great in relation to the paralysis as that which is seen in typical cases of myatonia congenita. Neither the absence of the deep reflexes nor the electrical reactions could aid in the differential diagnosis.

On the pathological side the evidence is strongly in favour of regarding the condition as primarily myelopathic. The loss and atrophy 
of the motor-cells of the ventral horns is very striking, and although similar changes have been described in a few cases of myopathy it has never been so severe, and in most cases no such atrophy of the spinal motor-cells is present. A similar diminution in the number and in the size of the cells has been found in the condition described by Oppenheim as myatonia congenita (Baudouin, Collier and Holmes), but it has not been nearly so severe as that which we found in this case.

It is, however, not altogether easy to confirm the view that in our case the disease is a primary myelopathic affection due to a degenerative process affecting the cells of the ventral horns, for no evidence of this degeneration in actual progress can be obtained either by the Marchi or by the Nissl method; though the latter method shows chromatolytic changes in a few cells, yet the essential feature is the smallness and normal appearance of such cells as are present. Even the muscle-changes cannot decide the question definitely, as it is now generally recognized that the histological differences between the primary and the secondary muscular atrophies are not so sharp and distinct as was at one time believed. Many of the changes which we have described in the muscle-fibres resemble closely those of the primary myopathies-smallness and atrophy of most of the fibres, increase in size and hypertrophy of others, central degeneration and fission of some of the hypertrophied fibres-and if we attempt to draw a distinction it must be in the relatively small amount of connective tissue that is found between the individual fibres, and in the absence or rarity of free muscle-nuclei, as compared with that which exists in dystrophic muscles. On the other hand, such features might be expected to occur if a dystrophy commenced at an exceptionally early age, when the development of the muscle-fibres is not completed or so advanced as at the age at which the disease usually sets in. It may be pointed out that the same relatively slight fibrosis of the affected muscles and rarity of free muscle-nuclei has been found in myatonia congenita, a disease which also sets in very early in life.

The whole question of the pathology of these cases of WerdnigHoffmann paralysis has been recently discussed by Batten in "Brain," 1911, and the cases recorded in the literature to that date are there given.

\section{REFERENCES.}

Batten. Brain, 1911, vol. xxxiii, p. 433.

Baudouin. La Sem. Med., 1907, t. xxvii, p. 241.

Collier and Holmes. Brain, 1909, vol. xxxii, p. 269. 